\title{
Quand le journaliste emprunte au publicitaire : " what else? » dans la presse d'actualité générale
}

\author{
Desnica, Mirta \\ Céditec, Université Paris-Est \\ desnica.mirta@gmail.com
}

\section{Introduction}

Si l'on pense aux anglicismes, ce sont sans doute des mots comme look, gloss, big bang, software et autres gadgets qui viennent à l'esprit. Mais ces substantifs ne reflètent que très partiellement l'état actuel des traces de l'anglais en français. Au moment de l'intensification des contacts avec l'anglais à l'heure de la mondialisation de la communication, les chercheurs travaillant sur les anglicismes en français ont pu faire le constat d'une diversification des types d'emprunts - emprunts sémantiques, emprunt d'autres classes de mots que les substantifs, alternance codique... - ce qui rend « une approche purement lexicale, focalisée sur le substantifs empruntés [...] dépassée » (Humbley, $2010: 41$ ).

Il suffit en effet de feuilleter un magazine féminin ou de visionner un programme de téléréalité mettant en scène des jeunes pour se rendre compte de la récurrence dans le français actuel des unités linguistiques en anglais qui ont le statut non pas d'un mot lexical mais d'un énoncé complet ${ }^{1}$. Une approche discursive s'impose alors.

Parmi les nombreuses questions que les anglicismes comme I love you! Next! Let's go shopping ! Oh my God! et So cute! peuvent soulever dans l'esprit du linguiste soucieux de décrire et d'analyser ce phénomène, celle de savoir par quelle voie ces énoncés ont pénétré dans la presse est rarement facile. Afin d'apporter des éléments de réponse à la question de la circulation de ce type d'anglicismes, nous avons choisi d'étudier ici l'énoncé what else?, attesté dans la presse féminine (Desnica, 2014), qui présente l'avantage de pouvoir par ailleurs être clairement identifié comme étant le slogan publicitaire de la marque Nespresso.

Le rôle de la publicité dans la diffusion des anglicismes est en effet souvent évoqué. L'internationalisation de la stratégie marketing d'un nombre croissant de marques, qui cherchent ainsi à construire une même image dans tous les pays où elles sont présentes, fait que celles-ci choisissent de proposer les mêmes noms de produits et les mêmes signatures partout. La loi de 1996 qui rend obligatoire la traduction en français «des mentions et messages en langue étrangère accompagnant une marque » reflète bien cette réalité. Et pour une marque qui agit au niveau mondial ou cherche à se donner une image internationale, le choix de l'anglais semble évident. D'après une étude portant sur le plurilinguisme dans la publicité, entre 6 et $10 \%$ des publicités parues dans la presse française en 1995 comportaient ainsi des éléments en anglais, tandis que pour les autres langues étrangères, cette proportion ne dépassait pas les $0,5 \%$ (Lemaire, $2000: 213-217$ ).

Au-delà des aspects pratiques que lui confère le statut de la langue véhiculaire internationale, l'anglais a, selon les professionnels de la publicité, l'avantage de jouir d'un charme tout a fait particulier auprès des Français :

Il y a encore une espèce de fascination pour l'anglais, plus naturel, plus moderne que le français, plus ancien, plus littéraire... une signature parait plus « sexy », en termes de sonorité, à l'écoute, quand elle est en anglais. (B. Raynert et A. Vanhelle de Team Senior chez Ogilvy \& Mather., cité dans Publicité et langue française. Bilan 2009, p. 14) 
Si bien que l'on retrouve aujourd'hui une profusion de slogans publicitaires en anglais, voire des spots télévisés entièrement en anglais, sous-titrés en français. Tel est le cas de la campagne publicitaire de Nespresso qui nous intéresse ici ${ }^{2}$.

En 2006, l'objectif de la marque était de s'imposer comme l'icône de l'excellence du café portionné. L'agence McCann Paris a alors créé le premier d'une série de spots télévisés destinés au marché européen et imaginés autour d'une idée centrale mêlant humour et auto-dérision : dans chacun des épisodes de la «saga », l'égérie de la marque, l'acteur George Clooney, s'efface face à la seule star qui compte, Nespresso. La désirabilité extrême de Nespresso, dépassant celle du beau Clooney, est synthétisée dans le slogan Nespresso, what else? qui clôture chaque épisode.

En France, les résultats son exceptionnels : la publicité est en tête du classement du prix Ipsos des meilleures campagnes télévisées pour l'année 2007 et elle reste sur le podium les deux années consécutives. L'ensemble de la "saga» est élue campagne télé préférée de la décennie auprès des Français. La notoriété de la marque progresse, ainsi que le nombre de ses adeptes : les membres du club de la marque sont entre 20 et $30 \%$ plus nombreux à l'issue de chaque épisode. ${ }^{3}$

La notoriété de la campagne publicitaire de Nespresso a-t-elle permis à what else? de pénétrer dans le discours journalistique? Si l'expression est reprise par les journalistes, de quelle manière et avec quel sens est-elle employée? Dans quelle mesure ces emplois reflètent-ils le contexte d'origine de l'expression, à savoir le discours publicitaire de Nespresso ? Voilà quelques questions auxquelles nous essaierons de répondre.

Pour ce faire, nous avons choisi d'analyser la presse d'actualité générale qui, contrairement à la presse féminine, a l'avantage de s'adresser à un public très large, de traiter un grand nombre de sujets et de refléter l'usage du français courant. La présence de l'énoncé dans ce type de presse sera donc un indice d'une diffusion plus large. La base de données Factiva nous a permis de constituer un corpus exhaustif d'occurrences de what else? dans plusieurs quotidiens nationaux (Le Figaro, Libération, La Croix) et régionaux (Le Parisien, La Charente Libre, Ouest France, Sud Ouest), ainsi que dans deux hebdomadaires (Le Point, Le Nouvel Observateur).

Nous avons écarté les occurrences où what else? participe d'un segment en anglais plus long du type What else is there to see? et ceux qui témoignent des reprises du slogan par d'autres que les journalistes (là où l'expression correspond par exemple au nom d'un salon de coiffure ou d'un site internet, à un autre slogan comme «Morano, what else ? », etc.) qui sont très intéressants pour l'étude de la circulation de cet énoncé mais dépassent le cadre de ce travail. Nous avons ainsi obtenu un ensemble de 118 occurrences en contexte. La première n'est parue qu'en avril 2007, ce qui semble confirmer notre hypothèse de travail selon laquelle what else? a fait son entrée dans le discours journalistique français à partir de la campagne publicitaire de Nespresso.

Après une brève présentation des propriétés morphosyntaxiques de ces unités linguistiques, la partie centrale de notre analyse portera sur les aspects énonciatifs liés à la représentation du discours autre et sur les valeurs sémantico-pragmatiques de what else? Nous adopterons un point de vue synchronique, tout en apportant ponctuellement quelques éclairages d'ordre diachronique.

\section{Propriétés morpho-syntaxiques}

Dans la majorité des cas, what else? représente une phrase typographique - délimitée par une majuscule à gauche et par un signe de ponctuation à droite - comme dans le slogan publicitaire « Nespresso. What else? », mais les cas où il apparaît à l'intérieur d'une phrase sont nombreux aussi. Il est alors très souvent en position détachée, séparé du reste de la phrase par des virgules (de 1 à 3 ) ou des tirets (4), et intervient soit en fin de phrase (1 et 2), soit dans une position plus centrale (3 et 4$)$ :

1. Danser en bronzant, what else ? (Ouest France, 16-7-2013)

2. On ne s'en lasse pas. On ne s'en lassera jamais d'ailleurs. Des Beatles, what else ! (La Charente Libre, 2-11-2013) 
3. ...l'austère mais très chic pamphlet politique « Good Night and Good Luck », de et avec George Clooney qui aura, what else ?, le mot de la fin. (Le Parisien, 134-2009)

4. On dîne d'une charlotte de tourteau, d'un suprême de volaille aux légumes d'antan - what else ? - devant un tableau moderne. (Le Figaro, 21-10-2010)

Son fonctionnement correspond alors à celui d'un adverbe de phrase ( 2 ' et $3^{\prime}$ ) ou encore à celui d'une incidente (2"' et 3 "') :

2' On ne s'en lasse pas. On ne s'en lassera jamais d'ailleurs. Des Beatles, évidemment / bien sûr!

2" On ne s'en lasse pas. On ne s'en lassera jamais d'ailleurs. Des Beatles, cela va de soi / qui d'autre? / de qui voulez-vous qu'il s'agisse d'autre?, etc.

3. ... de et avec George Clooney qui aura, évidemment / bien sûr, le mot de la fin.

3". ...de et avec George Clooney qui aura - peut-il en être autrement ? / il ne peut pas en être autrement / cela ne surprendra personne / à quoi d'autre s'attendre? / on ne pouvait s'attendre à rien d'autre, etc. - le mot de la fin.

Lorsqu'il est intégré à la relation prédicative, what else ? apparaît le plus souvent en position de nom commun. Ce fonctionnement syntaxique tient dans la plupart des cas à l'emploi de ce segment en mention, c'est-à-dire en tant que signe autonyme. Il est alors introduit typiquement par l'article défini et une épithète (5) :

5. ...le fameux « What else ?» avec George Clooney... (Sud Ouest, 20-1-2010)

5, ... le fameux énoncé / slogan, etc. avec George Clooney...

Mais le corpus comporte aussi quelques exemples d'emplois nominaux plus originaux :

6. La prestation aérienne de George Clooney n'étonnera personne, il fait quasiment du "What else?" (Le Nouvel Observateur, 23-10-2013)

7. ...un Don Clooney, au plus "Nespresso - what else ?" de son charme bullshit... (Libération, 10-2-2010)

Dans les deux cas, l'expression s'insère dans une locution. Dans (6), la construction dans laquelle il s'insère fait penser aux segments comme «faire du Marlon Brando », "faire du Hollande », etc. qui indiquent que l'on joue, ou que l'on se comporte, comme quelqu'un d'autre, à la manière de quelqu'un d'autre, qui est alors pris pour un personnage typifié. Dans cet exemple, où l'on pourrait paraphraser what else? de la façon suivante :

6' La prestation aérienne de George Clooney n'étonnera personne, il fait quasiment du George Clooney dans la publicité de Nespresso.

on dit de Clooney que dans le film en question (Gravity) il joue son propre rôle, celui qu'il a joué dans la publicité Nespresso. L'énoncé qu'il y prononce devient ainsi une métonymie du personnage même.

En revanche, dans (7), what else ? commute avec un adjectif dans un emploi nominal :

7' ...un Don Clooney, au plus haut / fort de son charme bullshit...

Il peut apparaître également en position de nom propre :

8. Monsieur "What Else ? » arrive quand même à s'en sortir avec les honneurs. (Le Parisien, 24-4-2008)

8' Monsieur Clooney / Dupont / Lemaire...

On retrouve ensuite un petit nombre d'occurrences où what else? occupe la place syntaxique d'un adjectif qualificatif (9).

9. $\quad$ L'effet "What else" ? (Le Figaro, 20-9-2010)

9' L'effet surprenant / attendu / incommensurable, etc.

Il convient pourtant de remarquer que la construction « effet + Nom » est également possible et récurrente dans le français actuel. On peut alors rapprocher cet emploi des lexies comme «l'effet boumerang / 
papillon / boule de neige / Doppler » ainsi que des «effet Morano / Mika / Big Brother » que l'on peut lire dans la presse et sur Internet.

Enfin, mentionnons aussi cette occurrence où what else ? commute avec un pronom (10) :

10. What Else demander de plus pour des vacances réussies ? (Libération, 4-7-2009)

10' Que / quoi demander de plus...

\section{Quelques aspects énonciatifs}

Si l'on admet que what else? a été diffusé à partir de la publicité de Nespresso qui l'a mis en circulation, on constate que dans la presse française cet énoncé est au moins doublement hétérogène (dans le sens d'Authier-Revuz 1984) : d'un côté, il constitue un élément en langue étrangère, et d'un autre côté, il renvoie à un discours autre que celui du journaliste.

Il est alors intéressant de se poser la question du marquage de ce «corps étranger » et des rapports qu'il entretient avec le discours journalistique en cours. Nous tâcherons d'y apporter quelques éléments de réponse en prenant appui sur l'appareil conceptuel élaboré par Jacqueline Authier-Revuz (1995, 2004) autour de ce qu'elle appelle la représentation du discours autre.

\subsection{Les modes de traitement de l'altérité}

Quelle image donne-t-on de cet acte d'énonciation autre que constitue what else? Comment se configuret-il au sein de l'acte d'énonciation en cours? Les exemples de notre corpus s'organisent de ce point de vue en deux grandes catégories : celle où l'on montre la matérialité des signes de ce discours autre (3.1.1) et celle où, tout en montrant des mots venant d'un autre, on en fait usage (3.1.2). Après avoir présenté ces deux modes de traitement de l'altérité de what else? dans le discours journalistique, nous dirons quelques mots sur une marque supplémentaire de l'altérité qui peut les accompagner : la paraphrase traduisante (3.1.3).

\subsubsection{Citer le discours d'un autre}

Le premier cas, qui concerne une trentaine d'occurrences, correspond à ce que l'on appelle habituellement la citation : on crée une représentation d'un acte d'énonciation autre par le moyen d'un segment autonyme. Le discours autre est alors signalé et délimité par des guillemets ${ }^{4}$ et attribué explicitement ou implicitement à un locuteur, s'agissant le plus souvent de l'acteur George Clooney. Les occurrences où What else? apparaît sous la forme du discours direct canonique, c'est-à-dire où celui-ci est introduit par un syntagme qui indique le locuteur de l'énoncé cité et comporte un verbe de parole (11), sont assez rares ; il s'agit plus souvent d'un discours direct sans syntagme introducteur (12) :

11. George Clooney qui dit «What else ? » aux buveuses de Nespresso, [...] c'est du Gondry. (Le Figaro, 1-3-2008)

12. Comme on ne peut absolument pas résister au charme de George Clooney, en dépit de son activisme publicitaire ("What else ?") en direction des caféïnomanes, on était tout frétillant à l'idée de voir Michael Clayton de Tony Gilroy. (Libération, 17-10-2007)

Mais bien plus souvent cet énoncé apparaît comme l'équivalent d'un nom, comme dans l'exemple (5). Comme nous l'avons déjà noté, les cas où il est précédé d'un article indéfini sont très rares. Lorsque l'énoncé est attribué à Clooney ou à Nespresso, cela se limite à cet exemple où l'on décrit en quelques mots le spot publicitaire et l'énoncé qui y apparaît. On notera que l'article en question a paru lors de la première année de la diffusion de la « saga » dans l'espace médiatique français :

13. Ses films les plus connus sont ses réalisations pour Air France, où défilent des nuages sur une musique planante des Chemical Brothers, et pour Nespresso avec un George Clooney dépité concluant le spot d'un ironique «What else ? ( Le Figaro, 5-3-2008) 
Dans les articles ultérieurs, plus besoin de raconter la publicité ni de qualifier l'énoncé selon sa valeur intradiscursive (l'attitude du personnage de Clooney lorsqu'il énoncé "What else? », le sens que cet énoncé a dans la publicité, etc.). Il devient alors typiquement :

$$
\text { 14. ...le « What else? » de George Clooney (Le Parisien, 8-1-2011) }
$$

La valeur de «connu » que véhicule l'article défini qui précède quasi systématiquement cet énoncé ${ }^{5}$ ne tient pas à un emploi anaphorique. En effet, il ne s'agit pas de faire référence au même énoncé mentionné précédemment dans le texte mais de poser cet énoncé comme faisant partie de la mémoire intertextuelle commune au journaliste et au lecteur. Ceci est renforcé par le choix d'épithètes comportant le trait sémantique de notoriété. On peut même observer un effet de gradation qui suit l'ordre chronologique :

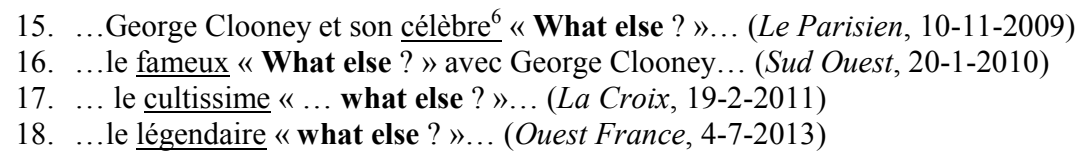

Le «What else ? » de Clooney semble être rentré dans la légende à côté des «Je pense, donc je suis » (Descartes), «I have a dream » (Martin Luther King), «C'est fin, c'est très fin, ça se mange sans faim » (Père Noël est une ordure) et autres aphorisations ce de type, dans le sens que D. Maingueneau (2012) donne à ce terme.

\title{
3.1.2 Présenter son discours comme affecté par un autre discours
}

Dans les emplois de cette catégorie, qui comporte près de 90 occurrences, l'acte d'énonciation d'origine ne constitue pas, comme plus haut, le référent du discours du journaliste; il «n'est plus ce dont on parle mais ce qui interfère dans le dire, ce qui l'altère $[. .$.$] en intervenant comme source [\ldots]$ de ses manières de dire » (Authier-Revuz, 2004: 44). On parlera alors de modalisation autonymique d'emprunt: on emprunte des mots à un autre tout en montrant qu'ils viennent d'ailleurs. L'énoncé qui en résulte fait ainsi entendre une deuxième voix à côté de celle du journal ; dans la terminologie de Bakhtine il est bivocal :

\begin{abstract}
[L]'auteur peut utiliser le mot d'autrui à des fins personnelles en dotant d'une nouvelle orientation interprétative ce mot qui possède déjà son orientation propre et ne la perd pas pour autant : ce mot doit encore être perçu comme mot d'autrui. Ainsi dans un seul mot peuvent se trouver deux orientations interprétatives, deux voix. (Bakhtine, $1970: 262$ )
\end{abstract}

Cette modalisation du dire peut être indiquée explicitement par un commentaire méta-énonciatif. Dans notre corpus, ceci se limite aux trois exemples suivants :

19. «What else » (quoi d'autre ?), comme le dirait George Clooney, l'ambassadeur de la marque. (Le Figaro, 11-4-2008)

20. "What else ? " comme dirait George Clooney. La PME spécialiste des matériaux plastique d'origine végétale se trouve également à la conception de la première bougie votive $100 \%$ biodégradable. (Sud Ouest, 13-5-2010)

21. What else ? comme on dit à Hollywood. (La Croix, 18-12-2008)

On constate que la source de cette manière de parler autre qui est ainsi indiquée est George Clooney et les gens d'Hollywood.

Bien plus souvent le journaliste ne juge pas nécessaire de signaler à qui il emprunte la manière de dire en disant «what else? ?. Le renvoi à un ailleurs est alors le produit d'un travail interprétatif du lecteur qui peut être sollicité par la présence des guillemets :

22. Doté d'un charme irrésistible, d'un humour décapant, d'une humilité sans faille, George Clooney est devenu l'un des acteurs les plus «bankables » de Hollywood. «What else? » (Le Point, 11-10-2007) 
Il s'agit alors de reconnaître ce segment comme faisant référence à un déjà-dit en puisant dans sa mémoire intertextuelle. C'est en effet grâce à la notoriété présupposée et effective de cet énoncé que le renvoi peut rester très souvent implicite et fonctionner comme une allusion :

23. "Vendre absolument en direct au consommateur en telle quantité, c'est unique au monde", note Denis Fages.

A quoi ça tient ? A George Clooney, what else?.', répondront en chœur les convertis. Et ce n'est pas faux. (Libération, 2-11-2007)

Ici, les mots mêmes utilisés permettent au lecteur de comprendre que le journaliste a repris le célèbre slogan publicitaire, le travail interprétatif étant d'autant plus aisé que l'article traite de Nespresso.

\subsubsection{Marquer le caractère autre d'un segment par la paraphrase}

Avant de continuer, quelques remarques concernant un phénomène qui peut marquer le caractère étranger d'un élément, la paraphrase, qui, dans le cas qui nous préoccupe ici, a la valeur d'une traduction. Les exemples ne sont pas nombreux et ne vont pas au-delà de la fin de l'année 2011. Ceci pourrait indiquer qu'après plus de cinq ans de présence de la publicité l'énoncé est considéré suffisamment connu et compris des lecteurs de sorte qu'il ne nécessite plus de traduction.

La paraphrase traduisante peut accompagner les deux types de représentation du discours autre: la citation et la modalisation autonymique d'emprunt. Elle se situe alors immédiatement après l'énoncé What else? $(24,25$ et 27$)$ ou un peu plus loin dans le texte (26). Elle est signalée par l'expression « en effet » (25) ou bien son repérage est purement interprétatif (24, 26 et 27) :

24. What else ? Quoi d'autre ? La fameuse question publicitaire de George Clooney, dont on n'a jamais vraiment su à qui elle s'adressait, vient de trouver deux réponses qui ne ravissent pas la multinationale suisse Nestlé et sa filiale Nespresso. (Sud Ouest, 15-5-2010)

25. Station de ski fermée! What else ? Quoi d'autre, en effet ? (Sud Ouest, 7-42008)

26. «Nespresso, What else? » susurre George Clooney dans les publicités pour le café en dosettes inventé par Nestlé. Quoi d'autre que Nespresso ? Les dosettes Casino ! (Le Figaro, 5-3-2010)

27. De la verdure, un amas de vieilles pierres qui blondissent sous le soleil et quelques arpents de vigne... What else ? Quoi de mieux en effet pour accomplir à peu de frais ce rêve universel de retour à la terre, embrasser le noble travail de vigneron sur fond de château restauré ? (Sud Ouest, 21-11-2008)

Dans les $70 \%$ des cas la paraphrase qui est proposée correspond à la traduction officielle figurant dans la publicité elle-même : «quoi d'autre ? »-c'est le cas de 24, 25, et 26 -, mais on trouve aussi quelques traductions plus libres, comme dans (27).

\subsection{Les modalités d'apparition du discours autre}

Après avoir vu les différentes formes que le marquage de l'altérité de what else? prend dans la presse française, intéressons-nous à présent aux modalités selon lesquelles cet énoncé autre peut surgir au sein du discours journalistique en cours. Le pivot de l'analyse sera constitué par la distinction que J. AuthierRevuz (1995: 317 et ss., 2010) fait entre discours autre attaché à l'objet (appelé initialement « approprié ») et discours autre associé :

A la différence du discours autre approprié, qui surgit dans le dire, comme le discours imposé par l'objet dont il est question, en tant qu'il est un attribut, un élément de cet objet, le discours autre associé apparaît, lui, s'opposant au premier de façon non discrète, comme surgi de l'interdiscours, du champs de forces et d'échos interdiscursifs que traverse le dire pour se faire, comme un discours autre, qui, spécifiquement 'présent' ou 'pressant' parmi tous les discours qui l'environnent s'impose au discours en train de se faire [...]. » $(1995: 330)$ 
Bien que cette opposition soit transverse au champ de la représentation du discours autre, nous nous limiterons ici aux cas relevant de la modalisation autonymique d'emprunt, qui nous semblent les plus intéressants à explorer sous cet angle-là. La question sera donc de savoir de quoi parlent les journalistes lorsqu'ils reprennent cet énoncé, c'est-à-dire en lien avec quels thèmes, quels référents (quels « objets de discours », dirait J. Authier-Revuz) il apparaît. Comme la frontière entre les deux versants n'est pas étanche, nous examinerons aussi quelques cas qui nous semblent appartenir à une zone intermédiaire.

\subsubsection{What else ? comme discours autre attaché à l'objet du discours en cours}

Dans le cas qui nous intéresse ici what else? apparaît comme "'trouvé' par le discours dans la réalité qu'il vise », ce dernier «fai[sant] place aux mots mêmes de ce dont il parle » (Authier-Revuz, 1995 : 337). Conformément à notre hypothèse de travail, what else ? sera considéré comme attaché à l'objet du discours journalistique lorsque celui-ci traite de Nespresso et en reprend le slogan.

En effet, un nombre intéressant d'occurrences apparaît lorsqu'on informe sur un nouveau produit de la filiale de Nestlé, comme les machines à café Zenius et Aguila dans (28), ou lorsqu'on apporte une information quelconque concernant la marque, comme par exemple l'ouverture d'une boutique (29) :

28. C'est fait, Zenius et Aguila, deux machines à café de la marque, ont déjà été présentées par Nespresso et le fournisseur d'accès Internet Orange. Scellant ainsi un partenariat original. Leur particularité est de communiquer, par une carte SIM, avec l'entreprise afin d'effectuer des diagnostics de maintenance à distance. La connexion permet également de faire une évaluation des stocks. Mais pour l'instant, ces systèmes M2M, (machine to machine) sont réservées aux seuls professionnels. What else? (Le Parisien, 24-2-2012)

29. What else ?

Evénement sur les Champs-Elysées. Le 18 décembre prochain, Nespresso ouvrira sa plus grande boutique-bar au monde, $1500 \mathrm{~m} 2$ entièrement dédiés au café, répartis en trois espaces : le Barista Bar, lieu de découverte et d'initiation ; le Lounge, pour se détendre autour d'une sélection de... cafés, et le Dressing-Room, l'endroit «trendy » de la boutique, un espace ludique où l'on pourra faire customiser sa machine Essenza dans seize coloris craquants. Ne manque plus que le beau George (Clooney). Dommage !

Nespresso : 119, avenue des Champs-Elysées, 75008 Paris. (Le Figaro, 3-112007)

Mais si what else? est sans aucun doute le signe de Nespresso, il semble l'être devenu encore plus pour l'acteur qui prête son image à la marque, son «ambassadeur», George Clooney. Un discours sur Nespresso mentionnera presque inévitablement Clooney, comme le montre l'exemple précédent. L'acteur est devenu l'emblème de la marque et «what else? » est devenu son propre emblème. Si bien qu'on le reprend - pour parler comme Clooney - lorsqu'il est question de l'acteur en lien avec son activité publicitaire liée à Nespresso :

30. Pendant un an, la jeune femme a récupéré 25000 capsules de Nespresso et les a minutieusement positionnées pour donner naissance à un portrait de l'égérie de la marque, en 9 mètres par 8 . S'il vient à survoler ce parking, l'acteur s'y posera peut-être pour proposer un café à la jeune femme. Eh, what else ? (Sud Ouest, 28-7-2012)

mais aussi lorsqu'on parle de lui dans un tout autre contexte : lorsqu'il pose à côté de sa moto Fat Boy (31), joue dans un film (32), s'engage politiquement (33)... :

31. Un Fat Boy, what else ? », George C., Los Angeles. (Le Figaro, 2-8-2010)

32. Pour vous accompagner lors de ce périple céleste, Sandra Bullock interprétera le docteur Ryan Stone, qui effectue sa toute première expédition dans l'espace. N'ayez pas peur, George Clooney, alias Matt Kowalsky saura vous guider. What else? (Le Nouvel Observateur, 4-11-2013)

33. A la veille du référendum sur l'autodétermination du Sud-Soudan, George Clooney s'est rendu à Juba pour soutenir la population locale. Au côté de John 


\begin{abstract}
Kerry, ex-candidat démocrate à la Maison-Blanche, l'acteur-messager de l'Onu a appelé les différentes mouvances à une sortie pacifique du conflit. La paix dans le monde, what else ? (Le Point, 20-1-2011)
\end{abstract}

Rappelons - en quittant un instant la présentation des emplois allusifs de what else? - que lorsque le slogan est cité, il est presque systématiquement attribué à Clooney comme nous l'avons déjà vu dans plusieurs exemples plus haut : c'est lui qui le dit; cet énoncé, c'est le sien. Et what else ? en est devenu l'étiquette au point de remplacer son nom, voire de devenir son deuxième prénom. Sous la plume de certains journalistes, 1'Américain devient ainsi " Monsieur 'What Else ?' » (Le Parisien, 24-4-2008) ou encore « George 'What else' Clooney » (Libération, 12-9-2011). De plus, lorsqu'il s'agit de rapporter un énoncé comportant les mêmes mots mais prononcé par quelqu'un d'autre, comme par exemple par le musicien Miles Davis, le discours de Clooney lié à Nespresso surgit aussitôt :

34. Les quelques minutes divulguées, sur une bande d'environ une heure et demie, témoignent d'une émotion palpable quand Miles fait son entrée contre toute attente dans le musée. D'abord nerveux, puis confiant et enfin amusé, allant même jusqu'à ôter ses éternelles lunettes noires pour se lancer sur le phrasé des trompettistes de Saint-Louis et terminer ses réponses par un "What else ?" qui ne doit rien à George Clooney. (Libération, 19-10-2009)

Selon la tonalité générale de l'article, le fait d'énoncer le slogan de la marque en parlant de Clooney ou de Nespresso permet d'attribuer des jugements appréciatifs ou dépréciatifs à l'acteur et la marque. Mais dans tous les cas, ce procédé, que nous avons proposé ailleurs de désigner par le terme " mimétisme » (Desnica, 2014), donne une image de proximité avec les sujets en question : en adoptant une expression qui leur est propre, on se montre au plus près d'eux, pour exprimer ainsi son admiration ou pour mieux s'en moquer.

\title{
3.2.2 Quelques cas intermédiaires
}

Il y a des cas où what else? n'est pas repris lorsqu'on thématise Nespresso ou son égérie et pour lesquels il nous semble problématique de parler de discours autre attaché à l'objet, mais où l'on perçoit quand même un lien avec la publicité en question. Comme il est d'ailleurs impossible de tracer avec exactitude une frontière qui séparerait l'espace discursif auquel un élément est propre de celui auquel il est étranger, nous avons décidé de faire apparaître dans notre analyse une zone intermédiaire.

A partir de la publicité pour le café portionné Nespresso, l'énoncé qui nous préoccupe semble être devenu signe du café en général. On le retrouve en effet à plusieurs reprises lorsqu'une information concerne le café en capsules non pas de Nespresso mais de l'un de ses concurrents (comme par exemple Jo Malone en 35), ou bien si l'article parle d'une autre enseigne de café (comme Starbucks, 36) :

\section{What Else ?}

Elle cherchait la note parfaite, celle du café fraîchement moulu le matin. 300 essais plus tard, Jo Malone dévoile Black Vetyver Café, un sillage corsé autour du grain de café noir, du vétiver et de l'encens. [...] 80 eur les $100 \mathrm{ml}$ au Bon Marché et dans les boutiques Jo Malone (Le Figaro, 15-3-2010)

36. Café chez Starbucks, «New York Times» gratos, what else ? (Libération, 1-32013)

mais aussi lorsqu'il est question de café en dehors d'un contexte commercial : des entretiens d'embauche organisés dans une cafétéria (37), le plaisir d'une pause café en terrasse (38), un fait-divers qui implique des distributeurs de boissons chaudes (39)... :

37. Un café pour un emploi, what else? (Le Parisien, 18-11-2011)

38. Le piéton a eu envie de crier « Alléluia! », hier midi, en retrouvant le plaisir simple, mais oublié, de la pause café en terrasse. Cinq minutes de pur bonheur ! Et nul besoin de voir sourire Georges Clooney pour apprécier l'instant. Un rayon de soleil généreux qui cajole les pommettes. L'entracte du concert entêtant des marteaux-piqueurs. Les éclats de rire et la bonne humeur des Sarladais. What else ? (Sud Ouest, 6-3-2010) 
39. Un café, what else ? Et la monnaie en plus. Un employé de maintenance de distributeurs de boissons chaudes a été braqué hier, à 11 heures, route de Vert-leGrand, à Echarcon. (Le Parisien, 7-5-2011)

Par métonymie, l'univers de référence de «what else ? » s'est donc étendu de Nespresso à l'univers de la commercialisation du café, au café en tant que tel et aux endroits et aux objets liés au café (cafétéria, distributeur de boissons chaudes, etc.).

Les occurrences de l'ensemble suivant représentent également une extension du domaine de pertinence de l'expression, cette fois-ci non pas par rapport à l'objet de la publicité mais par rapport à la personnalité de son acteur principal, et ce par analogie. En effet, what else? fonctionne parfois comme moyen de caractériser une attitude, un éthos proche de celui de l'acteur, celui d'un séducteur nonchalant à l'américaine :

40. Si Jarnac n'est pas Washington - et encore moins Hollywood - Arnaud Montebourg a pris des airs de George Clooney, mercredi et jeudi, sur les bords de Charente. En marge du congrès de la " C6R », le député de Saône-et-Loire s'est surtout plu à rappeler sa rencontre " formidââble » avec la " team » (équipe, NDLR) de Barack Obama, en début d'année. D'humeur badine, le politique aux lunettes noires a fait étalage d'un savoir-faire de génial communiquant « made in USA » - en mode estival et super-relax.

Sur le parvis de l'hôtel Renard - presque la Maison blanche -, le « rénovateur » gratifia notamment les observateurs d'un geste technique remarquable : sans laisser tomber son «I phone » de sa main gauche, il saisit au vol une petite fille blonde pour une photo souvenir du plus bel effet. Arnaud le trublion aussi fort que Barack, what else... (Sud Ouest, 29-8-2009)

L'attitude décontractée, familière (« en mode [...] super-relax »), les accessoires «branchés » (« lunettes noires », «I phone »), le langage affecté («sa rencontre 'formidââble' ») et anglicisé (« la 'team'»), la pose devant la caméra et d'autres éléments grâce auxquels il a fait étalage de son « savoir faire de génial communicant » ont fait reconnaître dans Arnaud Montebourg les airs de Clooney. Et un what else? surgit aussitôt.

Et comme le what else? publicitaire est prononcé par le célèbre Clooney qui y joue le célèbre Clooney, cet énoncé semble également être perçu, grâce à une généralisation par métonymie, comme appartenant au langage propre au monde du star-système américain (le lecteur se souviendra du commentaire métaénonciatif « comme on dit à Hollywood » cité plus haut, significatif à cet égard), aux célébrités et à la jet-set d'une manière générale, y compris le monde de la mode :

41. Et l'on dîne à la carte du Grand Véfour, avec aux fourneaux nul autre que le célèbre Guy Martin. Vincent Boeuf vous confiera même, entre deux bouchées de tarte aux truffes, qu'Elton John et Giorgio Armani seraient venus y fêter leurs anniversaires et qu'Arielle Dombasle y dînera le soir même. What else ? (Le Point, 12-8-2010)

42. Charlotte Casiraghi, Kate Moss et Elodie Bouchez ont fait la fête après avoir assisté au premier défilé de Chanel dans la capitale. " Paris-Londres », collection de " prêt-à-couture » créée par Karl Lagerfeld dans les ateliers d'art de la maison, a été présentée au son de la musique rock and roll de Sean Lennon, fils de John. What else ? (Le Point, 20-12-2007)

L'énoncé apparaît ensuite dans un nombre intéressant d'articles qui ont trait à la langue anglaise ou aux pays anglophones. Il ne souligne alors pas le lien avec la marque Nespresso mais s'affiche comme une expression anglaise, comme signe de la langue anglaise en tant que telle, et qui est aussi celle des publicités télévisées de Nespresso.

On trouve ainsi des what else ? qui agrémentent différents articles traitant de l'apprentissage de l'anglais (43) ou d'autres phénomènes liées à la Grande-Bretagne ou les Etats-Unis (44). En voilà quelques exemples : 
43. «Les cours seront dispensés par des professeurs expérimentés, de langue maternelle anglaise $»$, tient à souligner la compagnie de transport [SNCF]. Cerise sur le gâteau - ou le pudding -, cet enseignement est éligible au DIF (droit individuel à la formation) ou peut être pris en charge par la formation professionnelle continue. What else ? (Le Figaro, 19-10-2013)

44. Tout a en effet été pensé pour les amateurs de vin et de l'Union Jack. On croisera donc aux coins des rues de Vallet les enfants du cercle celtique de Clisson et les musiciens du Pipe Band écossais de Saint Herblain, avec le kilt à carreaux porté comme il le faut. Tandis que les Jaguar anglaises de l'old english car s'élanceront sur les routes du vignoble pour rencontrer les viticulteurs lauréats des derniers concours de vin. What else? (Ouest France, 16-3-2012)

Le dernier exemple mérite que l'on s'y attarde un peu. Il s'agit d'un extrait d'un article traitant du salon du vin Expo-Vall' imaginé cette année-là autour du thème «Le Royaume-Uni en muscadet ». Alors que les organisateurs de la manifestation ont créé l'ambiance britannique en saturant la décoration des lieux et l'offre gastronomique de symboles d'outre-manche - Union Jack, bus rouge, reconstitutions de devantures de pubs, faux soldats de la garde royale, Jaguar, kilt à carreaux, fish and chips, thé... -, le journaliste a essayé de rendre l'esprit du salon en parsemant son discours d'éléments en anglais. Le texte relatant cet événement " 'so british' » est ainsi ouvert par une allusion au titre de l'hymne britannique, détourné pour l'occasion («God save the Muscadet! ») dans le chapeau, et il est clôturé par un « what else? ?.

Enfin, il convient de mentionner ici les cas où l'énoncé intervient lorsqu'il s'agit d'informer sur des biens et des services présents sur le marché tout en les conseillant au lecteur. L'article prend alors l'allure d'une publicité. En voici juste un exemple ${ }^{8}$ :

45. Hormis les économies réalisées à la pompe, tout à bord rappelle la version classique. Côté confort et sécurité, doit-on encore narrer les mérites de cette compacte hyperconnue et ultra-adorée par la terre entière ? Rappelons que la Golf - deuxième modèle le plus vendu au monde - a célébré en juin son 30 millionième exemplaire... Chapeau bas ! On pourra donc retrouver les équipements et les aides à la conduite de sa cousine. Jolie finition, écran couleur tactile central, alerte à la somnolence, Bluetooth, commandes au volant ou radars de stationnement... What else ? (Le Point, 17-10-2013)

What else? vient clore une présentation fort positive de la nouvelle Golf BlueMotion. Le choix de cet énoncé qui fait entendre un slogan publicitaire serait-il une façon d'assumer, sur un registre ludique, la tonalité publicitaire du texte?

\subsubsection{What else ? comme discours autre associé au discours en cours}

Tout en gardant à l'esprit que l'opposition attaché/associé est en réalité un continuum, observons à présent quelques cas où l'objet du discours au sein duquel what else ? apparaît est très éloigné, voire sans lien apparent avec l'univers des publicités de Nespresso.

Même si les cotextes de what else? présentent alors un éventail assez large de thèmes et de domaines, quelques généralisations peuvent être faites.

En effet, on a eu recours à l'énoncé à plusieurs reprises lorsque l'article traitait d'une forme de loisir : le sport (et les plaisirs du farniente à la plage) (46), la musique (47), le cinéma (48), le restaurant, les videsgreniers, etc. :

46. À l'instar de Patrick Cintio, quatre autres moniteurs, parachutistes professionnels et brevets d'état, composent l'équipe de Wafou. Leur ambition? Ouvrir une école qui leur permettrait de proposer plus que du tandem. "Il nous manque l'autorisation de la communauté de communes », explique le parachutiste qui a bon espoir d'obtenir le feu vert pour 2009. En attendant, c'est l'autorisation d'atterrir sur la plage Pereire qui éclaire sa saison 2008. [...] « On saute, on atterrit. Les amis sont sur le bateau, on va prendre une coupe au Zen It et manger au Cap Pereire. » Effectivement... What else ? (Sud Ouest, 22-7-2008) 
47. Où bien alors, attendez un peu qu'il se mette au piano, son premier instrument, pour des escales un peu plus jazz et rock. Là aussi son jeu fait mouche. Philippe Grancher l'accompagne d'une voix qui colle à son (ses) style(s) sur des reprises ou des compos des plus léchées. Du vrai blues, au gros son, bien carré et bon à entendre. Pour clôturer cette édition, what else ? (La Charente libre, 9-7-2011)

48. «What else? » En dehors de longues séquences hallucinatoires, on aura droit tout de même à une petite scène d'avortement et à des incursions dans le corps humain aussi sexy qu'un scanner. Après le cinéma-vérité, saluons l'avènement du cinéma coloscopique. (Le Figaro, 23-5-2009)

Deuxièmement, on le retrouve dans deux articles proposant des conseils pratiques dans le domaine de la maison et de la famille : l'exemple (49) rapporte les astuces d'une adepte du nettoyage écologique et (50) donne des « idées pour survivre au trajet avec des enfants en bas âge » :

49. Peu de casseroles résistent à Danièle... « On met juste un peu d'eau de Javel. On fait chauffer, en ouvrant bien les fenêtres à cause du chlore. Puis, avec une spatule en bois, on frotte et ça s'en va tout seul. » Mais attention : cette astuce ne convient pas pour les casseroles munies d'un revêtement antidérapant ou les Cocottes-Minute. «Pour celles-ci, on met quelques gouttes de liquide vaisselle avec de l'eau très chaude et on laisse tremper... ». What else ? (Le Parisien, 141-2013)

50. Un moment donné l'enfant a faim. Oui car l'enfant est un ventre. Il faut toujours avoir dans sa boite à gants, juste à côté de l'éthylotest, une boite de gâteaux ou un paquet de bonbons. Éviter ceux au chocolat car ils fondront et créeront le drame. - Le résultat: l'enfant a la bouche (et l'esprit) occupé pendant 54 secondes (temps de mastication).

- Le risque: l'obésité (Lien: leplus.nouvelobs.com/contribution/761312-enfantsobeses-il-faut-modifier-la-facon-dont-les-parents-voient-le-surpoids.html). What else? (Le Nouvel Observateur, 3-8-2013)

Avec les deux sous-ensembles précédents, on peut voir une certaine continuité avec l'univers de la consommation : les loisirs en question sont une forme de consommation culturelle et les textes mêmes sont souvent empreints d'hédonisme, l'un des registres privilégiés de la publicité ; (49) rappelle les démonstrations que l'on peut voir par exemple dans les émissions de télé-achat, et, tout comme (50), adopte la forme et le ton des articles de la presse féminine ( 10 idées/astuces pour...»), qui est ellemême une forme de loisir intrinsèquement liée à la consommation. Mais toutes les occurrences ne peuvent pas être expliquées comme déclenchés par ces idées-là, comme le montrent les exemples suivants qui traitent respectivement d'une recherche universitaire portant sur le chant du coq et de pèlerins venus célébrer les Journées mondiales de la jeunesse :

51. Le professeur Takashi Yoshimura, de l'université Nagoya (Japon. What else ?), s'est intéressé quant à lui au chant du coq : pourquoi le pousse-t-il dès potronminet, comment l'a-t-il appris, etc. (Sud Ouest, 7-4-2013)

52. Tous ces étrangers venus emplir la salle Lauga de leurs accents chantants et lointains constituaient les quelque 1200 pèlerins accueillis dans le diocèse de Bayonne dans le cadre des Journées mondiales de la jeunesse qui s'ouvrent aujourd'hui à Madrid. What else ? (Sud Ouest, 16-8-2011)

Si l'énoncé reste un symbole de Nespresso et de son égérie, on voit que l'étendue des contextes où il est susceptible d'apparaître sous la plume d'un journaliste est considérable, notamment à partir de la deuxième année de sa présence dans la presse. Certaines occurrences, apparues dans des contextes très éloignés de celui de l'univers de la publicité, invitent à se demander s'il s'agit encore là d'une allusion au célèbre slogan ou si, au contraire, l'expression n'y est pas perçue par le journaliste comme un « simple » emprunt à la langue anglaise. 


\section{Valeurs sémantico-pragmatiques}

What else? c'est donc Nespresso et George Clooney, c'est une attitude particulière, c'est la jet-set, c'est l'anglais, et pas seulement. Mais que signifie cet énoncé pour les journalistes? «Quoi d'autre?», répondraient-ils sans doute, conformément à la traduction officielle. Mais ce segment, qui d'ailleurs ne peut pas commuter avec toutes les occurrences de notre corpus, n'en est pas moins polysémique. Il nous reste donc à présent de nous interroger sur les valeurs sémantico-pragmatiques de what else ?, autrementdit sur son sens en contexte.

Nous nous limiterons ici aux occurrences syntaxiquement autonomes (ou relativement autonomes) qui sont de loin les plus fréquentes, même si l'interprétation des segments tels que "l'effet 'What else?", «il fait pratiquement du 'What else?' » et «au plus 'Nespresso - what else ?' de son charme » mentionnés plus haut soulèvent d'autres questions intéressantes.

\subsection{What else ? 1: « évidemment! »}

L'emploi qui est le plus en accord avec le sens que cet énoncé prend dans la campagne publicitaire, et qui est aussi le plus fréquent, est celui où l'on propose une réponse négative à la question donnée en surface : «Quoi d'autre? - Rien d'autre!» La structure interrogative ne correspond donc pas ici à une question véritable - il ne s'agit pas de demander à l'allocutaire de fournir une information -, mais à ce qu'on appelle une question « rhétorique » qui « énonce une assertion par le biais d'une pseudo-interrogation, en écartant toute possibilité de choix » (Ducard, 2004 : 77). La réponse étant présélectionnée par le locuteur, il ne reste à l'allocutaire qu'à « confirmer par un acquiescement ce que pense le locuteur » (Moignet, cité dans Kerbrat-Orecchioni $1991: 25)$.

Les occurrences de what else? de ce type, que nous désignerons par what else?1, permettent la glose suivante : étant donné les préconstruits (dans le sens de Pêcheux 1990), cela ne pouvait être rien d'autre I on ne pouvait s'attendre à rien d'autre, etc. Cet énoncé peut alors commuter avec « évidemment ».

Avant de passer aux exemples journalistiques, regardons de plus près le dialogue publicitaire dans lequel cet énoncé apparaît pour la première fois :

53. Camilla (s'approchant de George qui vient de se servir un café et le déguste devant la machine à café Nespresso) : Excuse me, I'm sorry, do you mind if I... George (il cherche dans ses poches) : Of course! I don't think I have a pen... Camilla: Huh? (pause) Oh, I just want a Nespresso. George: Yeah. Oh. (pause) Nespresso, what else? ${ }^{9}$

On pourrait gloser de la façon suivante : «Etant donné l'excellence du café Nespresso (un préconstruit affiché par la marque, celui qu'elle vise à construire dans la population), ce que la jeune femme demandait ne pouvait être rien d'autre qu'un Nespresso, il ne fallait s'attendre à rien d'autre ». Camilla voulait un Nespresso et non pas un autographe de Clooney, évidemment.

Retournons maintenant à what else ? 1 dans notre corpus journalistique :

54. Contre une telle force, la bande d'« Ocean's 13 » résiste courageusement : 99104 complices après une première semaine à 280492 pour Paris/périphérie et 871 826 pour la France entière, c'est OK pour George Clooney. What else ? (Le Point, 5-7-2007)

55. Jean Chalon parle de Louise de Vilmorin : what else ? (Le Figaro, 31-1-2013)

Dans le premier extrait, what else?1, qui peut ici être paraphrasé aussi par «Pourrait-il en être autrement? », "Comment s'en étonner? », etc., indique que vu la popularité de Clooney, son nouveau film ne pouvait être qu'un succès. Dans le deuxième, il nous apprend que le fait que J. Chalon parle de L. Vilmorin n'a rien de surprenant : « de qui voulez-vous qu'il parle d'autre ? (l'écrivain est l'auteur de sa biographie). ${ }^{10}$ 
Nous avons voulu savoir s'il s'agissait là d'une expression courante en anglais ou d'une invention de Nespresso. Pas de mention de what else? dans les dictionnaires de référence de la langue anglaise que nous avons pu consulter : le syntagme n'y figure pas parmi les expressions idiomatiques. Néanmoins, une recherche rapide dans la base des données de la presse anglophone nous a permis de constater que cet emploi de what else ? est bien attesté. Il a pourtant été intéressant de remarquer que cette expression ne s'y construit pas de la même manière que dans la presse française. En effet, dans cette dernière, what else ?1 apparaît systématiquement après le segment sur lequel porte le jugement de l'évidence. Cette position est également attestée dans la presse anglophone, mais les occurrences où celui-ci est antéposé sont de loin plus nombreuses. En voici juste un exemple :

56. Donald Trump bet on Virginia wines two years ago, buying Kluge Estate winery and naming it - what else? - Trump Winery... (The Washington Post, 24-112013)

Un argument de plus pour étayer l'hypothèse selon laquelle le what else? français s'est construit d'après la publicité Nespresso.

\subsection{What else ? 2: " on ne pourrait rien demander de mieux ! »}

L'ensemble d'emplois suivant partage certains traits avec le précédent. Tout comme what else?1, what else ?2 a la valeur d'une question rhétorique, appelant une réponse négative à la question en surface, qui est cette fois-ci : «Que demander de mieux, de plus ? - Rien ! ». La glose est alors la suivante : on ne pourrait rien demander/espérer d'autre/de mieux/de plus, ou encore : c'est tout ce qu'il faut (pour être heureux) :

57. Ce chouette bistrot dispose d'une grande terrasse dans une rue piétonne. Cuisine généreuse, service pêchu et prix abordables. What else ? (Le Nouvel Observateur, 29-7-2013)

Les occurrences ayant cette valeur apparaissent souvent après une énumération de faits positifs, qui correspondent souvent à des attributs d'un produit, d'un service, etc. Dans l'exemple qui précède, il s'agit d'un restaurant alliant «cuisine généreuse, service pêchu et prix abordables ». Le what else ?2 qui suit demande en apparence ce que l'on pourrait demander de plus, ou de mieux, pour inviter le lecteur à s'identifier à la réponse induite : « rien ! ${ }^{11}$

Parfois l'énumération des qualités d'un objet ou d'un fait ne précède pas immédiatement what else ?2 mais une liste de ces qualités peut être établie au fur et à mesure de la lecture de l'article. What else ?2 arrive alors comme la conclusion de cette description-argumentation comme l'équivalent d'un «ça y est, on (vous et moi) est convaincu! ». C'est le cas de l'exemple suivant :

58. La machine Aguila : dernière-née des machines à café Nespresso Business Solutions, elle allie les meilleures caractéristiques de la méthode traditionnelle des baristas aux dernières innovations signées Nespresso. Dotée de quatre têtes d'extraction, cette machine dédiée à l'hôtellerie de luxe (elle coûte $10000 €$ et équipe en France une vingtaine d'établissements gastronomiques et de luxe) prépare simultanément par simple pression d'une touche des cafés et autres recettes chaudes ou froides à base de mousse de lait. Elle est également connectée par une carte Sim directement au siège, afin de prévenir des éventuels dysfonctionnements avant même qu'ils n'apparaissent. What else? (Le Parisien, 21-11-2011)

Une machine qui allie la tradition aux dernières innovations, avec un grand nombre de têtes d'extraction, adoptée par les établissements de luxe, multifonctionnelle, on ne peut plus simple à utiliser et capable de signaler au siège de Nespresso des dysfonctionnements avant même qu'ils apparaissent, que de pourraiton imaginer de mieux, en effet!

C'est sans doute le détachement de what else ? au sein de la publicité même de Nespresso qui a facilité l'évolution du sens de l'expression dans cette direction-là. En effet, à notre connaissance, l'énoncé ne 
constitue une réplique du personnage de Clooney que dans deux spots : celui dont il a été question et un deuxième construit autour d'un quiproquo similaire. Il s'agissait là encore d'un what else?1. Ultérieurement, il apparaîtra uniquement à la fin de chaque publicité, en dehors des dialogues, en tant que slogan de la marque prononcé en voix-off: Nespresso. What else? L'accent peut alors être mis sur l'aspect «il n'y a rien de mieux (que Nespresso) », ce qui induit, nous semble-t-il, l'apparition dans la presse de what else ?2 à partir de la fin de l'année 2008.

\subsection{What else ? 3 : une question}

Les exemples qui suivent actualisent le sens littéral que l'expression a dans une question et ont ainsi la valeur d'une question véritable. Plusieurs sous-ensembles peuvent être dégagés ici. La variante la plus fréquente est celle où l'on demande à quelqu'un de rajouter un élément à la liste établie précédemment, d'indiquer la suite d'une description, d'un processus, etc. :

59. La Corse, Londres... «What else ?» Thierry Ligonnière n'en fait pas mystère. « On y travaille ! [...] ». (en parlant des nouveaux vols saisonniers de l'aéroport de Quimper) (Ouest France, 5-9-2012)

60. Musique lounge, éclairage soigné, capsules de café exposées en vitrines comme des bijoux... and what else ? Georges Clooney en vitrine bien sûr! (Sud Ouest, 3-2-2010)

Des vols saisonniers relient déjà Quimper à la Corse et à Londres. «Qu'est-ce qui suit ? ", «Quelle est la destination suivante ? », demande-t-on dans le premier exemple. Le deuxième exemple est très intéressant d'un point de vue énonciatif. Avec « and what else? » on demande au lecteur de compléter la description de la boutique tout en lui donnant un indice. Le lecteur et le journaliste répondent d'une voix : "Georges Clooney en vitrine bien sûr ! »

Ou bien what else ?3 est une manière de demander quels sont les autres choix disponibles, comme dans les exemples 24 et 26 vus plus haut.

Mais parfois la question est auto-adressée pour devenir une manière de représenter son activité énonciative en cours :

61. En Suisse, où le système fonctionne depuis un an, la machine fait un carton. Et les people de l'Hexagone se l'arrachent déjà. Ils seront d'ailleurs nombreux, ce soir, au happening de lancement parisien. What else? Ah oui, BabyNes promet de vous doser le biberon parfait en une seule petite minute avec de l'eau filtrée grâce un code-barres qui libère la quantité exacte d'eau nécessaire à la préparation. (Le Parisien, 6-9-2012)

En se montrant comme son propre récepteur, le journaliste se demande : «que dire d'autre? » ou bien «qu'est-ce que je allais dire d'autre? » à l'aide d'un what else?3 dialogique sur le plan intralocutif (Bres, $2005: 53)^{12}$.

Enfin, une valeur exclamative peut s'ajouter à cette question portant sur la suite d'un phénomène. En voici un exemple :

62. CYRANO A bien ri en constatant que certaines personnes confondaient l'Office de tourisme de la ville avec un cyber-café. Hier, Cyrano a observé que depuis que l'antenne située dans la rue Neuve-d'Argenson avait mis à la disposition de sa clientèle un accès libre à Internet, de plus en plus de gens passaient des heures à surfer sur le web avec leur propre ordinateur. Et ce n'est pas tout. Il a aussi appris que certains visiteurs allaient même jusqu'à demander aux jeunes hôtesses de leur servir un café. What else? (Sud Ouest, 14-8-2010)

«Et ce n'est pas tout » et «aussi » signalent l'ajout d'un nouveau élément à l'exposé de Cyrano, encore plus incroyable que le précédent. Le développement, que l'on pourrait paraphraser ainsi : «Un nombre croissant de personnes utilisent les locaux de l'Office de tourisme pour surfer sur Internet pendant des heures ; pire encore, certains n'hésitent pas à demander aux hôtesses de leur servir un café ", induit à 
interpréter le what else? final comme l'équivalent d'un « Et puis quoi encore ? », "Qu'est-ce qu'il (ne) va (pas) leur falloir de plus? », «Ça va aller jusqu'où? ». Il fonctionne alors comme une question à valeur exclamative exprimant l'indignation, à la manière d'un « Vous vous rendez compte ?! ».

Signalons pour terminer que le sens de certaines occurrences reste ambigu. Dans le texte portant sur le salon du vin par exemple (43), what else ? est-il une marque de l'évidence ? (Mais qu'est-ce qui serait évident dans ce cas ?). Traduit-il au contraire la surprise ? Pose-t-il une question sur la suite ? (La suite de quoi ?)... Est-il bien autre chose que l'affichage d'une expression anglaise ?

\section{Conclusion}

A la suite de la présentation des résultats de cette étude exploratoire de l'utilisation de what else ? dans la presse française d'actualité générale, nous pensons avoir montré que l'expression anglaise, diffusée à partir du slogan de Nespresso, est devenue usuelle dans l'écriture journalistique. Phrase autonome ou incidente, adverbe de phrase, nom commun ou nom propre, pronom, la variété syntaxique des emplois est large. Tout comme celle des effets de sens qu'elle acquiert et des contextes dans lesquels elle apparait. Emblème de Nespresso et de George Clooney, son égérie, elle semble être devenue aussi, par un effet d'extension de l'univers de référence, le signe du café, des séducteurs "à la Clooney », du monde du show-business, de la langue anglaise... Si elle porte aujourd'hui de fortes connotations « Nespresso », la fréquence de ses emplois en lien avec des phénomènes tout autres et des emplois où c'est son caractère anglais qui s'affiche laisse envisager que what else? rentre dans les années à venir dans le français ordinaire en tant qu'une expression courante empruntée à l'anglais, c'est-à-dire une expression «branchée ».

Mais un tout autre scénario peut également être envisagé. Le contrat de Clooney avec Nespresso ayant expiré fin 2013, la marque sera peut-être amenée à engager un(e) autre ambassadeur(drice). Que deviendra le what else? français si le célèbre slogan est rattaché à une autre tête d'affiche et prononcé dans un contexte différent, ou encore s'il est remplacé par un autre slogan ? Le temps montrera si l'expression s'installera durablement dans le discours journalistique et dans la communication spontanée ou s'il s'agissait juste d'une expression à la mode, vouée à disparaître avec la campagne publicitaire qui l'a mise en circulation.

\section{Références bibliographiques}

Authier-Revuz, J. (1984). Hétérogénéité(s) énonciative(s). Langages, 73, 98-111.

Authier-Revuz, J. (1995). Ces mots qui ne vont pas de soi. Boucles réflexives et non-coïncidences du dire, 2 tomes. Paris : Larousse.

Authier-Revuz, J. (2004), La représentation du discours autre : Un champ multiplement hétérogène, Lopez-Muñoz, J.-M., Marnette, S., Rosier, L. (dir.), Le discours rapporté dans tous ses états : question de frontières, Paris L'Harmattan, 35-53.

Authier-Revuz, J. (2010). Variations autonymiques dans les Mémoires de Berlioz : les mots sur le devant de la scène. Richard, E. et al (éd.), Aux marges des grammaires. Rennes : Presses universitaires de Rennes, 61-75.

Bakhtine, M. (1970). La Poétique de Dostoïevski, traduit du russe par Kolitcheff, I. Paris : Editions du Seuil.

Bres, J. (2005). Savoir de quoi on parle : dialogue, dialogal, dialogique, dialogisme, polyphonie... Bres, J. et al. (dir), Dialogiqme et polyphonie : approches linguistiques. Actes du colloque de Cerisy. Bruxelles : De Boeck, 47-61.

Desnica, M. (2011). Le fonctionnement discursif des énoncés fashion dans la presse féminine française contemporaine. Le rôle des énoncés fashion dans le texte et dans la construction de la représentation discursive des acteurs de la communication, mémoire de master dirigé par C. Olivier, Lyon : Université Jean Moulin Lyon 3.

Desnica, M. (2014 à paraître). «Parler l'autre»: de l'expressivité des énoncés fashion dans la presse féminine française, Pavelin-Lešić, B. (dir), Francontraste : l'affectivité et la subjectivité dans le langage, Mons : CIPA. 
Ducard, D. (2004). Une discussion biaisée : la question rhétorique dans le débat parlementaire, Ducard, D., Entre grammaire et sens, Etudes sémiologiques et linguistiques. Paris : Ophrys, 73-88.

Humbley, J. (2010). Peut-on encore parler d'anglicisme ? Lexique, normalisation, transgression : actes du colloque du 7 septembre 2010, Université de Cergy-Pontoise. Limay : Mes mots, 21-45.

Kerbrat-Orecchioni, C. (dir) (1991). La Question. Lyon : PUL.

Lemaire, S. (2000). Le plurilinguisme dans la publicité. Göttingen : Cuvillier Verlag.

Maingueneau, D. (2012). Phrases sans texte. Paris : Armand Colin.

Pêcheux, M. (1990). L'inquiétude du discours: textes choisis et présentés par D. Maldidier. Paris : Editions des Cendres.

Publicité et langue française. Bilan 2009., Autorité de régulation professionnelle de la publicité, Rapport d'étude commandée par le Ministère de la Culture et de la Communication, novembre 2009.

\footnotetext{
${ }^{1}$ Pour une analyse des formes et des emplois de ce type d'anglicismes dans la presse féminine, voir notre mémoire de master (Desnica, 2011).

${ }^{2}$ C'est aussi le cas par exemple de la publicité télévisée actuelle pour le parfum Dolce \& Gabbana The One avec Scarlett Johansson et Matthew McConaughey et de celle pour la Citroën DS5 avec Ewan McGregor.

${ }^{3}$ D'après «Etude de cas : Nespresso, what else ? Les clés de succès de la pub TV préférée des Français », article mis en ligne le 24 avril 2011 sur les pages du Syndicat national de la publicité télévisée, http://www.snptv.org/etudes/etudes-de-cas-detail.php?id=20, consulté le 3 décembre 2013.

${ }^{4}$ Le statut autonymique des signes peut être signalé aussi par des italiques qui s'ajoutent parfois aux guillemets ou s'y substituent. Comme Factiva ne tient malheureusement pas compte des italiques dans les textes originaux, cette marque typographique ne pourra pas être incluse dans notre analyse.

${ }^{5}$ On retrouve aussi quelques exemples du type « George Clooney et son célèbre 'What else ?' » (Le Figaro, 25-52012) dont la paraphrase présuppose l'article défini : « le célèbre 'What else ?' de George Clooney ».

${ }^{6}$ Tous les soulignements sont de nous.

${ }^{7}$ Comme nous l'avons déjà signalé, la base de données que nous avons utilisée ne permet pas de savoir si dans les exemples de ce type what else? porte des italiques qui peuvent être une marque de la modalisation autonymique. Il n'en reste pas moins que, comme les guillemets, ce signe est polysémique : il peut aussi marquer l'autonymie pure, l'emprunt à une langue étrangère, le soulignement, une certaine intonation, etc. Même si l'énoncé était en italiques, le renvoi à un déjà-dit ne serait pas explicite mais le fruit d'un travail interprétatif du lecteur.
}

${ }^{8}$ Voir aussi l'exemple (57).

${ }^{9}$ Le sous-titrage en français :

Camilla : Excusez-moi, je suis désolée... Est-ce que je peux...

George : Bien sûr. Je crois que je n'ai pas de stylo...

Camilla : Je veux juste un Nespresso.

George : Nespresso. Quoi d'autre?

${ }^{10}$ Les exemples (2), (3), (4), (22), (23), (31), (32), (33), (40), (41), (42), (49), (50) et (51) vus plus haut comportent ce même emploi.

${ }^{11}$ Voir aussi les exemples (1), (27), (38), (45), (46) et (47).

${ }^{12}$ Voire aussi l'exemple (48). 